\title{
Diboson production (CDF)
}

\author{
Viviana Cavaliere ${ }^{* \dagger}$ \\ University of Siena - Via Roma 56, Siena (Italy) \\ INFN Pisa - Largo B. Pontecorvo 3, Pisa (Italy) \\ E-mail: viviana.cavaliereepi.infn.it
}

WW and WZ production in $p \bar{p}$ collisions at $1.96 \mathrm{TeV}$ are studied in samples of $\sim 3 \mathrm{fb}^{-1}$ of data using leptons, jets and missing $E_{T}$. Fully leptonic decays as well as semi-leptonic decays are measured. Diboson production is expected in the standard model, and predicted cross sections are confirmed. It is important to investigate various signatures as associated production of Higgs bosons is topologically similar.

European Physical Society Europhysics Conference on High Energy Physics July 16-22, 2009

Krakow, Poland

\footnotetext{
* Speaker.

${ }^{\dagger}$ for the CDF Collaboration
} 


\section{Introduction}

Diboson production is of great interest because it provides unique opportunity to test the Standard Model at the TeV scale, it constitutes a very important background to Higgs and SUSY searches and it is a probe to new physics through deviations of Triple Gauge Couplings (TGCs) from Standard Model predictions. In this paper we examine diboson production in $1.96 \mathrm{TeV} p \bar{p}$ collision using the CDF detector, compare this production to Standard Model predictions, and set limits on the strength of some anomalous couplings. Signals of the WW, WZ, ZZ are searched through their leptonic and semi-leptonic final states.

\section{Diboson production in leptonic final state}

\subsection{WW cross section and TGC}

We measured the WW production cross section in the two charged lepton ( $e$ or $\mu)$ and two neutrino final state using an integrated luminosity of $3.6 \mathrm{fb}^{-1}$ [1]. This measurement makes use of matrix element based likelihood ratios. A per-event probability is assigned according to a matrix element based calculation (MCFM) of the leading order cross section for 4 different processes (WW, $\mathrm{ZZ}, \mathrm{W} \gamma$, and $\mathrm{W}+\mathrm{jet}$ ) given the measured event kinematics. A likelihood ratio $\left(L R_{\mathrm{WW}}\right)$ is then formed using the event probabilities for signal and background like events. The WW cross section is the extracted using a binned maximum likelihood method which best fits $L R_{\mathrm{WW}}$ signal and background shapes to data (see Fig. 1). A total of 654 candidate events are observed with an expected background contribution of $320 \pm 47$ events. The measured WW cross section is $12.1 \pm 0.9$ (stat. $)_{-1.4}^{+1.6}$ (syst.) pb, that is in good agreement with the Standard Model prediction and represents the most precise measurement up to date.

We also set limits on the trilinear gauge couplings of the WW production. The analysis examines the $p_{T}$ distribution of the lepton with higher $p_{T}$ and does a fit in order to determine limits on the couplings. The HISZ parameterization [2] is used to make 3 independent parameters $\left(\lambda^{\mathrm{Z}}=0\right.$, $g_{1}^{Z}=\kappa^{\gamma}=1$ in Standard Model). We set the limits $-0.17<\lambda^{\mathrm{Z}}<0.17,-0.26<\Delta g_{1}^{\mathrm{Z}}<0.35$, and $-0.68<\Delta \kappa^{\gamma}<0.77$ where $\Delta g_{1}^{Z}$ and $\Delta \kappa^{\gamma}$ are the deviations of the parameters from the Standard Model values.

\subsection{WZ cross section and TGC}

To search for WZ diboson production we look for events where both bosons have decayed leptonically. Because this is a small branching fraction, in order to observe a signal we use an improved lepton selection that increases the acceptance. The analysis is made using $1.9 \mathrm{fb}^{-1}$ [3] and requires 3 electrons or muons plus $E_{T}$ (missing transverse energy). We find a total of 25 events where $21.6 \pm 2.6$ were expected. This gives a cross section of $4.3_{-1.0}^{+1.3}$ (stat.) \pm 0.2 (syst.) \pm 0.3 (lum.) $\mathrm{pb}$, compared to a theoretical cross section $\sigma_{\mathrm{NLO}}=3.7 \pm 0.3 \mathrm{pb}$. We then use the $p_{T}$ of the $\mathrm{Z}$ from WZ events to test the WWZ vertex. We consider the anomalous triple gauge couplings $\lambda, \delta g$, $\delta \kappa$ as defined in [4] and [5] and implemented in MCFM. In the Standard Model all three of these couplings are zero. We set the limits for $\Lambda=2.0 \mathrm{TeV},-0.13<\lambda<0.15,-0.15<\Delta g<0.24$ and $-0.82<\Delta \kappa<1.27$. 


\section{3 $\mathrm{ZZ}$ cross section and TGC}

This analysis is based on a data sample corresponding to $1.9 \mathrm{fb}^{-1}$ of integrated luminosity [6]. We look in two channels, one of which both the Z's decay to electron or muons and one in which one of the two $\mathrm{Z}$ decays in two neutrinos. We find $3 \mathrm{ZZ} \rightarrow \ell \ell \ell \ell$ candidates with an expected background of $0.096_{-0.063}^{+0.092}$ that gives a significance of $4.2 \sigma$. This is combined with the $\mathrm{ZZ} \rightarrow \ell \ell v v$ channel which used a matrix element discriminator to separate $\mathrm{WW}$ and $\mathrm{ZZ}$ and extract a $1.2 \sigma$ significant signal. The combined significance is $4.4 \sigma$. The measure cross-section is $1.37_{-0.58}^{+0.74}$ (stat.+syst.) pb which should be compared to the Standard Model value of $1.4 \mathrm{pb}$ at NLO.

The simultaneous production of two $\mathrm{Z}$ bosons from triple gauge coupling $\mathrm{ZZZ}$ and $\mathrm{ZZ} \gamma$ is not permitted by Standard Model. CDF has set limits based on $1.9 \mathrm{fb}^{-1}$ on these anomalous couplings, described in terms of parameteres $f_{4}^{\mathrm{Z}}, f_{4}^{\gamma}, f_{5}^{\mathrm{Z}}, f_{5}^{\gamma}$, in the channel where one of the $\mathrm{Z}$ decays to electrons or muons and the other $\mathrm{Z}$ decays hadronically to jets. The dijet mass spectrum is fit in the high $p_{T}$ region of the $\mathrm{Z}$ to constrain potential contributions from anomalous couplings. No excess $\mathrm{ZZ}$ production is found, therefore we can set the limits for $\Lambda=1.2 \mathrm{TeV},-0.12<f_{4}^{\mathrm{Z}}<0.12$, $-0.13<f_{4}^{\gamma}<0.12,-0.10<f_{5}^{Z}<0.10,-0.11<f_{5}^{\gamma}<0.11$.

\section{Diboson production in semi-leptonic final state}

Diboson production has been observed at the Tevatron in the lepton channels through the leptonic decays of the electroweak gauge bosons. Doing the same thing with jets is much more difficult due to the large background from $\mathrm{V}+\mathrm{jets}(\mathrm{V}=\mathrm{Z}, \mathrm{W})$. We present here the measurement of the cross section of VV decaying into two neutrinos plus jet and of WW/WZ into lepton-neutrino plus jets, that leads to the first observation of this signatures in both channels.

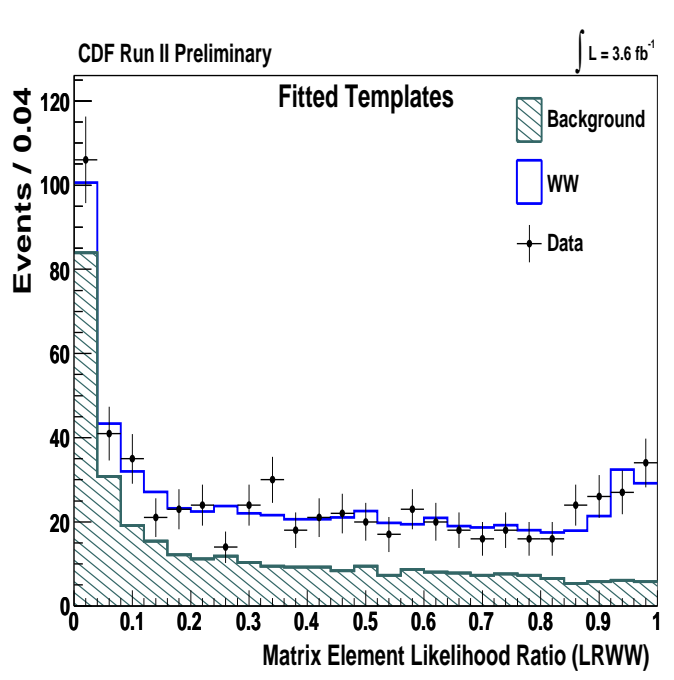

Figure 1: The $\mathrm{LR}_{W \mathrm{~W}}$ distributions for the signal $\left(\mathrm{W}^{+} \mathrm{W}^{-}\right)$and background processes after a maximum likelihood fit to the data.

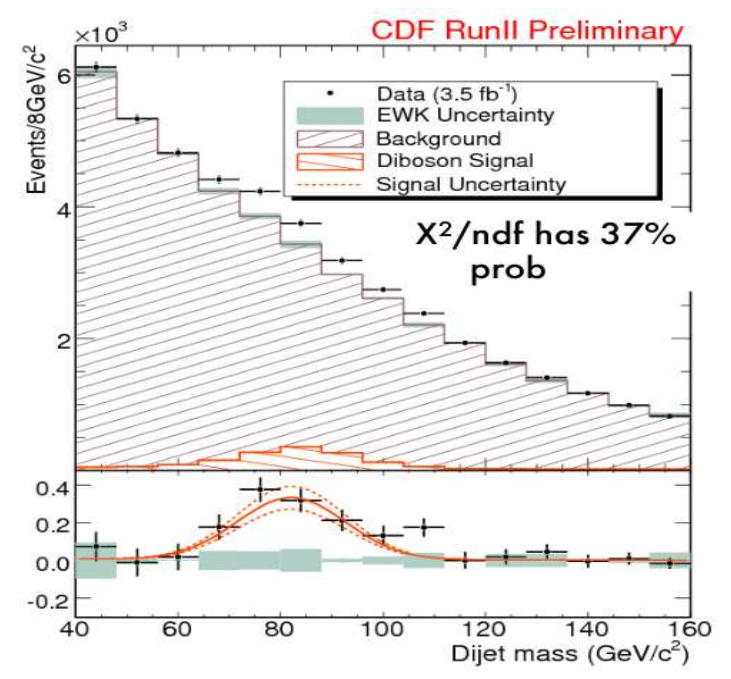

Figure 2: Signal extraction fit for $\mathrm{VV} \rightarrow \mathscr{E}_{T}^{\prime}+$ jets. The fit uncertainty (brown band) includes systematic uncertainties on shapes of QCD and EWK backgrounds. 


\subsection{VV in met+jets}

We measured the diboson cross-section using events with large $E_{T}(>60 \mathrm{GeV})$ and two jets with $E_{T}$ above $25 \mathrm{GeV}$. Due to limited energy resolution we cannot distinguish between WW, $\mathrm{WZ}$ and $\mathrm{ZZ}$ events so what we measure is really a sum of all these processes in our selection window. No cut on number of leptons in the event is performed therefore we are also sensitive to lepton decays of the gauge bosons. The QCD contribution, which is large in this channel, is heavily suppressed through novel algorithms related to $E_{T}^{\prime}$ significance. We extract the signal from the background using the invariant mass distribution of the two jets in the event (see Fig. 2). The extraction of the signal does not use the theoretical calculation of the $\mathrm{V}+\mathrm{jet}$ integral cross section and its invariant mass shape is cross checked with $\gamma+$ jets events from the data, hence considerably reducing the systematic uncertainty on the shape of this main background. We observe $1516 \pm 239$ (stat.) \pm 144 (syst.) that leads to a significance of $5.3 \sigma$ and we measure a cross section of $\sigma(p \bar{p} \rightarrow V V+X)=18.0 \pm 2.8$ (stat.) \pm 2.4 (syst.) \pm 1.1 (lum.) pb, in good agreement with the Standard Model expectations.

\subsection{WW/WZ in lepton-neutrino+jets}

We describe a search for WW/WZ $\rightarrow \ell v j j$ processes. Two different approaches are used.

The first one uses a data sample corresponding to approximately $3.9 \mathrm{fb}^{-1}$ of integrated luminosity to reconstruct WW/WZ events [8]. The diboson signal is extracted from the background using a $\chi^{2}$ fit of the invariant mass distribution, $M_{j j}$, of the two leading jet separately for the electron and muon samples. This simple method allows to search for a signal peak over a smooth background. The fit is performed in the $M_{j j}$ region from 36 to $200 \mathrm{GeV} / \mathrm{c}^{2}$ and estimates the fractions of signal, QCD and EWK backgrounds using $M_{j j}$ templates obtained from the CDF full simulation (signal and EWK) and data (QCD). The total EWK contribution is a free parameter of the fit while the relative normalizations of the processes forming the EWK background $(\mathrm{V}+$ jets and top) are fixed to the corresponding SM expectation. The QCD contribution is gaussian constrained to the value previously obtained from the MET fit. The fit (see Fig. 3 ) gives $1079 \pm 232$ (stat.) \pm 86 (syst.) $\mathrm{WW} / \mathrm{WZ} \rightarrow \ell v j j$ events, corresponding to a statistical significance of $4.6 \sigma$ ( $4.9 \sigma$ expected). We also measure $\sigma(\mathrm{WW} / \mathrm{WZ})=14.4 \pm 3.1$ (stat.) \pm 2.2 (syst.).

The other approach uses $2.7 \mathrm{fb}^{-1}$ and takes advantage of a multivariate technique to exploit all the information in the event [9]. We calculate event probability densities under the signal and background hypotheses using a set of measured variables of each event (the 4-vectors of the lepton and the two jets). The probability is constructed by integrating over the parton-level differential cross-section, which includes the matrix element for the process, the parton distribution functions, and the detector resolutions. We use the probabilities to construct a discriminant variable for each event, referred to as the Event Probability Discriminant, or EPD. To quantify the WW+WZ content in the data, we perform a binned maximum likelihood fit to the data (see Fig. 4). We fit a linear combination of signal and background shapes of the event probability discriminant to the data. The background normalizations (except for $\mathrm{W}+\mathrm{jets}$ that is a free parameter) are Gaussian constrained in the fit. Pseudo-experiments were carried out to determine the probability (p-value) that a background fluctuation would produce the observed excess. The median expected p-value was found to be $2.1 \cdot 10^{-7}(5.1 \sigma)$, whereas the observed p-value was $3.5 \cdot 10^{-8}(5.4 \sigma)$. The measured cross 


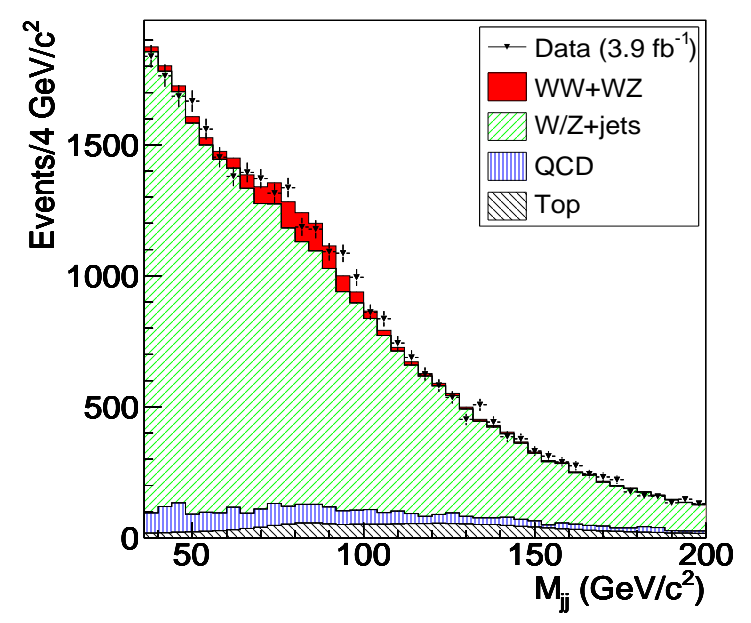

Figure 3: Dijet invariant mass distribution of reconstructed $\mathrm{W} / \mathrm{Z} \rightarrow j j$ candidates compared to the fitted signal and background components.

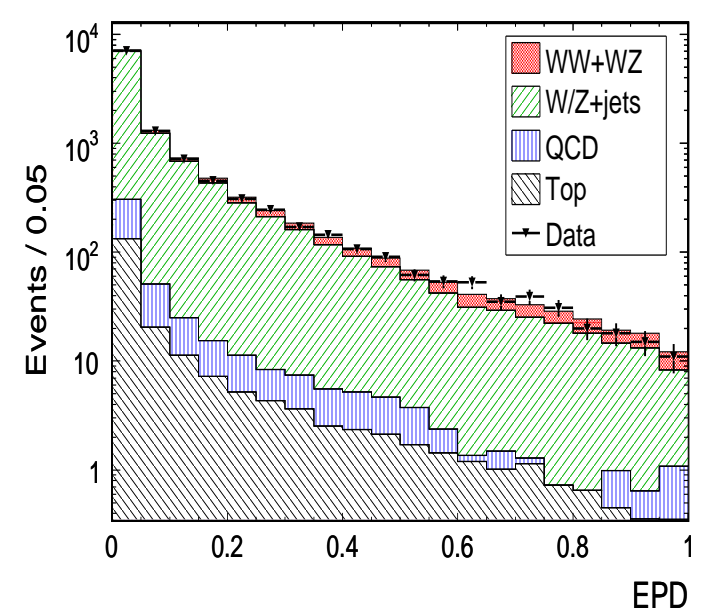

Figure 4: Observed EPD distribution superimposed on expected distribution from simulation

section is $17.7 \pm 3.9 \mathrm{pb}$. The results from the two approaches are in agreement with each other and with the Standard Model prediction $(16.1 \pm 0.3 \mathrm{pb})$.

\section{Conclusion}

We have studied diboson production in $p \bar{p}$ collisions and have found it to be consistent with Standard Model predictions. We have set limits on a number of different couplings that do not appear in the Standard Model. Finally we are now observing processes with cross sections less than a factor 10 larger than the Standard Model Higgs cross section.

\section{References}

[1] CDF Collaboration,http://www-cdf.fnal.gov/physics/ewk/wwllll/index.html

[2] K.Hagiwara, S. Ishihara, R. Szalapski and D. Zeppenfeld, Phys. Rev. D 48, 2182 (1993).

[3] A. Abulencia et al, Phys. Rev. Lett 98, 161801 (2007).

[4] J.M. Campbell and R.K. Ellis, Phys. Rev. D 60113006 (1999),

[5] R.K. Ellis, Nucl. Phys. B 160 (2006).

[6] T. Aaltonen et al, Phys. Rev. Lett 100, 201801 (2008).

[7] CDF Collaboration, http://www-cdf.fnal.gov/physics/ewk/2008/zzZatGC/ZzZaTGC.html

[8] CDF Collaboration, http://www-cdf.fnal.gov/physics/ewk/2009/jjlnu/index.html

[9] CDF Collaboration, http://www-cdf.fnal.gov/physics/new/hdg/results/wwwz_061909/ 